\title{
Grundlagen der autoradiographischen Darstellung der Nucleinsäuren in Gewebsschnitten mit Hilfe von Radio-Phosphor*
}

Von Eberhard Harbers und Karlheinz Neumann

Aus dem Institut für medizinische Physik und Biophysik der Universität Göttingen

(Direktor Prof. Dr. E. Witte) und dem Anatomischen Institut der Universität Göttingen

(Direktor Prof. Dr. E. B lech schmid t)

(Z. Naturforschg. 9 b, 175-180 [1954]; eingegangen am 9. November 1953)

Mit chemisch-analytischen und Zählrohrmethoden wurden die Grundlagen für eine selektive autoradiographische Darstellung der Desoxyribonucleinsäure (DNS) untersucht. Dabei stellte sich heraus:

1. Die Vorstellung, daß nach üblicher Vorbehandlung der Gewebsschnitte die Restaktivität von vorher verabfolgtem Radio-Phosphor ausschließlich den beiden Nucleinsäurefraktionen zuzuschreiben sei, ist unzutreffend.

2. Weder durch Behandlung der Gewebsschnitte mit Ribonuclease, noch alkalische oder saure Hydrolyse, noch durch die Kombination dieser Verfahren gelingt es, ohne Beeinträchtigung der DNS die übrigen Phosphor-Fraktionen vollständig zu extrahieren.

3. Die schwerwiegendste Fehlerquelle bei der selektiven Autoradiographie der DNS ist die Anwesenheit von Phospho-Proteinen, deren Radioaktivität die der DNS um ein Vielfaches übertreffen kann.

4. Es ist möglich, diesen Fehler durch Vergrößerung des Zeitraumes zwischen der Verabfolgung des ${ }^{32} \mathrm{P}$ und der Tötung des Versuchstieres weitgehend einzuschränken.

\section{I.}

$\mathrm{B}$ ei Stoffwechseluntersuchungen am Gewebsbrei ist die Zuordnung der interessierenden Vorgänge an bestimmte Gewebsstrukturen im allgemeinen unmöglich. Gerade dies aber schien uns als Ergänzung zu z. Zt. laufenden Nucleinsäure-Umsatzbestimmungen in Mäusetumoren mit Radio-Phosphor besonders wichtig. Wenn hierzu autoradiographische Verfahren herangezogen werden, so führt dies zu hohen Ansprüchen an die Methodik, da die in der Photoemulsion ausgelöste Schwärzung nur auf denjenigen Anteil des radioaktiven Phosphors zu beziehen sein soll, der vom Organismus in Nucleinsäuren eingebaut wurde. Praktisch bedeutet dies, daß sämtliche Phosphat-Fraktionen - bis auf die der Nucleinsäuren - aus dem Schnitt zu extrahieren sind, bevor er mit der Photoemulsion zusammengebracht wird. Fernerhin war es wünschenswert, durch weitere Extraktion auch der Pentosenucleinsäure $(=\mathrm{PNS})^{* *}$ selektiv nur die Desoxypentos e nucleinsäure (= DNS) autoradiographisch darzustellen.

* Die Untersuchungen wurden mit Mitteln der Deutschen Forschungsgemeinschaft durchgeführt.

** Da heute noch nicht sicher entschieden ist, ob die Nucleinsäuren keine anderen Zucker enthalten als Ribose bzw. Desoxyribose, sind von biochemischer Seite die allgemeineren Bezeichnungen Pentosenucleinsäure (statt Ribonucleinsäure) und entspr. Desoxypentosenucleinsäure eingeführt worden.
Einige Methoden, die dies ermöglichen sollen, sind in der Literatur beschrieben. So wurde von Leblond, Stevens und Bogoroch ${ }^{1}$ die PNS in den Gewebsschnitten durch Ribonuclease enzymatisch zerlegt und anschließend extrahiert. Von $\mathrm{How}$ a rd und $\mathrm{Pelc}^{2}$ sowie von $\mathrm{T}$ a y lor ${ }^{3}$ wurde - allerdings an pflanzlichen Objekten - die PNS mit $n$. HCl bzw. 10-proz. Perchlorsäure extrahiert. Eine kritische Nachprüfung, ob nach dieser Behandlung die im Schnitt verbliebene Radioaktivität wirklich nur oder wenigstens überwiegend auf die DNS zurückzuführen sei, wurde an tierischem Material bisher nicht vorgenommen. Uns schien eine solche Nachprüfung dringend erforderlich, da zahlreiche Befunde von biochemischer Seite, vor allem von D a vid s on und Mitarbb. ${ }^{4,5}$, darauf schließen ließen, daß außer den Nucleinsäuren noch verschiedene andere phosphat-haltige Substanzen im Gewebe verbleiben, deren sehr hoher Gehalt an Radio-Phosphor fälschlich den Nucleinsäuren zugeschrieben wird.

1 C. P. Lebond, C. E.Stevens u. R. Bogoroch, Science [New York] 108, 531 [1948].

2 A. Howard u. S. R. Pelc, Exp. Cell Res, 2, 178 [1950].

3 J. H. Taylor, Exp. Cell Res. 4, 164 [1953].

4 J. N. Davidson, S. C. Frazer u. W. C. Hutchison, Biochem. J. 49, 311 [1951].

5 J. N. Davidson u. R. M. S. Smellie, Biochem. J. 52, 594 [1952]. 
II. M ethodik: Versuchstiere waren Meerschweinchen, Wistar-Ratten und erbreine Mäuse der Stämme C 57 black und C $3 \mathrm{H}$. Untersucht wurden vor allem Leber, Milz und Tumoren (Crocker-Sarkom bei C 57 black und verschiedene Carcinome bei $\mathrm{C} 3 \mathrm{H})$. Abgewogene Gewebsmengen (200 bis $600 \mathrm{mg}$, je nach Organ) wurden entweder als Gewebshomogenat aufgearbeitet entspr. der nachstehend beschriebenen Technik von $\mathrm{L}$ a vik ${ }^{6}$ oder fixiert, eingebettet und histologisch weiterbehandelt, wie in den folgenden Abschnitten mitgeteilt wird.

a) Quantitative Nucleinsäure-Analysen: Zur Isolierung und Analyse der beiden NucleinsäureFraktionen wurde die Methode von Schmidt und $\mathrm{Th}$ annhauser ${ }^{7}$ in der Modifikation von $\mathrm{Lavik}$ und Mitarbb. ${ }^{6}$ benutzt. Dabei werden aus dem Gewebe zunächst die sog. säurelösliche Phosphat-Fraktion mit eisgekühlter 10-proz. Trichloressigsäure und anschließend die Phospholipoide mit Alkohol und Äther-Alkohol extrahiert. Danach werden die Gewebsreste mit $n$. KOH 18 bis 20 Stdn. lang bei $37^{\circ} \mathrm{C}$ exponiert. Die PNS wird dabei hydrolysiert, während die DNS in der Polynucleotidform erhalten bleibt. Durch Säurefällung läßt sich dann die DNS von der PNS, die in Lösung bleibt, trennen. Die quantitative Bestimmung erfolgte nach Veraschung durch kolorimetrische Phosphatanalyse nach G o m o r i ${ }^{8}$. Bei Versuchen mit Radio-Phosphor wurde außerdem an aliquoten Mengen mit einem Zählrohr die Aktivität bestimmt; der Quotient gezählter Teilchen pro mg P ergab dann die spezifische Aktivität der betr. Fraktion ${ }^{9}$. Es wurden im allgemeinen wenigstens 3 Parallel-Analysen durchgeführt; der mittlere Fehler der Einzelwerte lag um $10 \%$ bei den Untersuchungen am Gewebsbrei, stieg gelegentlich bis zu $25 \%$ bei der quantitativen Auswertung der histochemischen Reaktionen, die naturgemäß eine größere Streuung aufwiesen. Die außer den beiden NucleinsäureFraktionen im Gewebsrückstand enthaltenen Phosphat-Verbindungen (vor allem Phospho-Proteine $=\mathrm{PP}$ ) wurden nicht isoliert. Infolgedessen sind alle Analysenwerte für die PNS etwas zu hoch, da sie

6 P. S. Lavik, H. Harrington u. G. W. Buckalo o, Western Res. Univ., Atomic Energy Med. Res. Proj., Unclassified Docum., NYO-1625 [1951].

7 G. S chmidt u. S. J. Thannhäu ser, J. biol. Chemistry 161, 83 [1945].

8 G. Gomori, J. Lab. clin. Med. 27, 955 [1941].

9 E. H a r bers, Klin. Wschr. [im Druck].

* Die Versuche zur Gefriertrocknung wurden durch die Leybold-Hochvakuum-Anlagenbau G.m.b.H., Köln-Bayerıthal, gefördert.

10 K. Neumann u. E. Harbers [im Druck]. die Summe von PNS + PP wiedergeben. Während in der Originalarbeit von $\mathrm{S} \mathrm{chmidt}$ und $\mathrm{Th}$ a n n $\mathrm{h}$ a u s e ${ }^{7}$ noch angegeben wird, daß die Menge dieser phosphor-haltigen Verbindungen in den meisten Geweben vernạchlässigbar klein sei, haben neuere Untersuchungen ergeben, daß sie bei Versuchen mit Radio-Phosphor die Analysenwerte der PNS beträchtlich verfälschen können.

b) Quantitative Untersuchung der histochemischen Reaktionen: Die Fixierung der frisch abgewogenen Gewebsstücke erfolgte meist in C a r n o y scher Lösung, zum Teil durch Gefriertrocknung*. Die Analysenwerte von Gewebsbrei und gefriergetrocknetem Gewebe deckten sich gut, während durch Fixation in Carnoy vor allem PNS, aber auch etwas DNS, verlorenging ${ }^{10}$. Die Carnoysche L.ösung wurde im allgemeinen bevorzugt, da so während der Fixation schon ein Teil der ersten beiden Phosphat-Fraktionen (säurelösliche und P-Lipoide) extrahiert wurde. Die Gewebsblöcke wurden mit dem Mikrotom jeweils völlig aufgeschnitten. Die Schnittdicke betrug $20 \mu$. Da der Schnitt von beiden Seiten dem Lösungsmittel zugänglich ist, entspricht dies etwa einer Schnittdicke von $10 \mu$ beim Aufkleben auf den Objektträger. Alle Schnitte wurden dann gemeinsam den verschiedenen histochemischen Reaktionen ausgesetzt. Zur analytischen Auswertung wurde im allgemeinen die Fraktionierung entspr. der S chmidt-Thannha us er-Methode angeschlossen, in einigen Fällen das ganze Gewebe zur Phosphat-Analyse und Zählrohrmessung verwandt.

c) Hydrolyse mit Ribonuclease: $\mathrm{Zu}$ den Versuchen mit Ribonuclease wurde das kristallisierte salzfreie Ferment benutzt ${ }^{* *}$. Bei den makrochemischen Untersuchungen an aufgeschnittenen Gewebsblöcken erwies sich $1 \mathrm{mg}$ des Enzyms in $10 \mathrm{ml}$ Puffer-

** Das Präparat wurde von der Worthington Biochemical Sales Co., Freehold/New Jersey, USA, bezogen. Eine wesentliche Voraussetzung beim Ribonuclease-Test ist die Reinheit und Spezifität des benutzten Fermentes. Nach der Methode von McDonald ${ }^{11}$, die eine Verfeinerung des von $\mathrm{Kunitz}^{12}$ entwickelten Verfahrens darstellt, läßt sich Ribonuclease aus Rinderpancreas so darstellen, daß sie praktisch frei von Proteasen ist $\mathbf{1 3}$ und weniger als $1 / 50000$ an Desoxyribonuclease enthält. Letzteres ist wichtig, da die Extraktion der PNS unter unbedingter Schonung der DNS erfolgen soll. Die sehr sorgfältig durchzuführende Isolierung der kristallisierten Ribonuclease und ihre Testung auf ausreichende Reinheit ist in den meisten Laboratorien nur schlecht durchführbar und hat in den USA zu der allerdings recht kostspieligen industriellen Herstellung des Fermentes geführt.

11 M. R. M c D on ald, J. gen. Physiol. 32, 39 [1948].

12 M. Kunitz, Science [New York] 90, 112 [1939].

13 M. R. M cDonald, J. gen. Physiol. 32, 33 [1948]. 
lösung (0,1-m. Veronal-Acetat-Puffer, $\left.p_{\mathrm{H}} 6,8\right)$ als ausreichende Konzentration; exponiert wurde bis $\mathrm{zu}$ 12 Stdn. bei $37^{\circ} \mathrm{C}$. Zur Einwirkung auf histologische Schnitte wurde das Ferment in 0,01-proz. Lösung in McIlvaines Citronensäure-Phosphat-Puffer ${ }^{14}$ angesetzt; Expositionszeit maximal 4 Stdn. bei $37^{\circ} \mathrm{C}$. Die weitere Extraktion der säurelöslichen PhosphatFraktion mit Trichloressigsäure und der Phospholipoide erfolgte erst nach der Ribonuclease-Behandlung (vgl. Tab. 1).

d) Chemische Nucleinsäure-Extraktion aus Gewebsschnitten: Von verschiedenen Autoren wurde versucht, statt mit der schwer beschaffbaren Ribonuclease mit Säuren oder Alkali die PNS im Gewebsschnitt zu hydrolysieren und zu extrahieren. Als erste hatten Ericks on, Sax und Ogur ${ }^{15}$ festgestellt, daß sich aus pflanzlichen Objekten (MaisKeimlingen) durch 18-stdg. Exposition in 10-proz. Perchlorsäure bei $4^{\circ} \mathrm{C}$ die PNS extrahieren ließ, ohne daß dabei die DNS angegriffen wurde oder sichtliche proteolytische Effekte auftraten. Die Methode wurde aus einem speziell für pflanzliche Objekte geeigneten Verfahren zur Isolierung der Nucleinsäuren entwikkelt ${ }^{16}$ und damit zugleich makrochemisch auf ihre Brauchbarkeit geprüft. Von K ö n i g ${ }^{17,18,19}$, di S te fano ${ }^{20}$ und $\mathrm{Sulkin}{ }^{21}$ wurde die PerchlorsäureExtraktion an tierischen Geweben erprobt und als brauchbar befunden. Getestet wurde mit Farbreaktionen und UV-Absorptionsmessungen (Erhaltung der DNS im Zellkern). Für das Gelingen der Reaktion spielte die vorangegangene Gewebsfixation eine Rolle; ferner verhielten sich die verschiedenen Gewebsarten nicht gleichartig, sondern erforderten unterschiedlich lange Extraktionszeiten. Von Stich ${ }^{22}$ und $\mathrm{F}$ i s h e r ${ }^{23}$ wurden statt der Perchlorsäure Mineralsäuren (Salzsäure, Salpetersäure, Schwefelsäure) benutzt. Sulkin ${ }^{24}$ extrahierte die PNS aus dem Gewebsschnitt mit 0,1-n. KOH.

Die Testung dieser histochemischen Verfahren erfolgte in der in Abschnitt b) geschilderten Weise.

e) Autoradiographische Technik: Die Fixierung des Gewebes erfolgte in $\mathrm{C}$ a rn o y scher Lö-

14 R. E. Stowell u. A.Zorzoli, Stain Technol. 22 51 [1947].

15 R. O. Erickson, K. B. S ax u. M. Ogur, Science [New York] 110, 472 [1949].

16 M. Ogur u. G. Rosen, Arch. Biochem. 25, 262 [1950].

17 H. K ö n i g, J. nat. Cancer Inst. 10, 1346 [1950].

18 H. König u. H. Stahlecker, J. nat. Cancer Inst. 12, 237 [1951].

19 H. König u. H. S t a hlecker, Proc. Soc. exp. Biol. Med. 79, 159 [1952].

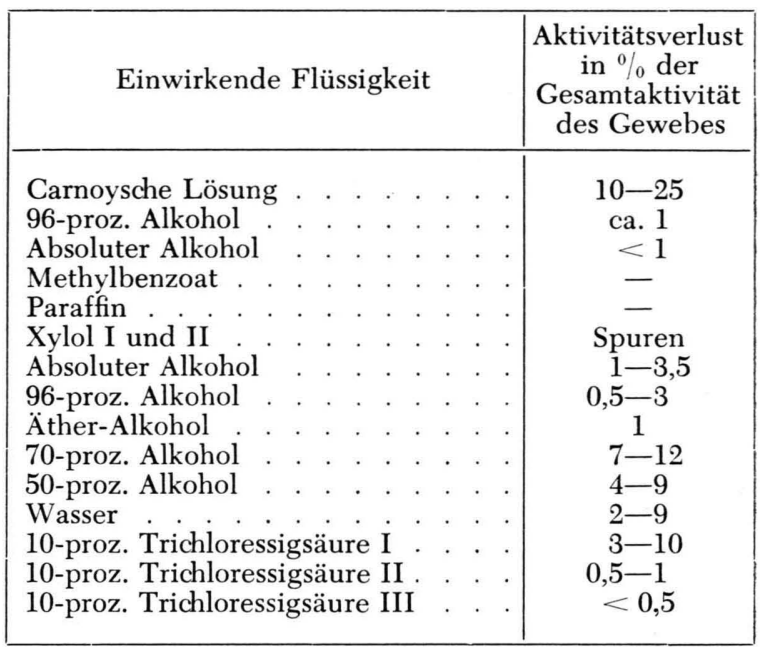

Tab. 1. Verluste von radioaktivem Phosphor bei der histologischen Vorbehandlung von Gewebsstücken und Schnitten (Leber, Milz, verschiedene Mäusetumoren). Das unterschiedliche Ausmaß der Verluste hängt von der jeweiligen Gewebsart, vor allem jedoch von der Zeitspanne zwischen der Injektion des Radio-Phosphors und der Tötung des Versuchstieres ab.

sung oder durch Gefriertrocknung ${ }^{25}$. Nach Entparaffinierung in Xylol, absteigender Alkoholreihe und Wässern wurden die Schnitte für $4 \mathrm{Stdn}$. bei $37^{\circ} \mathrm{C}$ in 0,01-proz. gepufferter Ribonuclease-Lösung eingestellt. Anschließend wurden sie 3-mal je $10 \mathrm{~min}$ mit 10-proz. Trichloressigsäure behandelt (vgl. dazu Tab. 1); danach wurden die Schnitte gründlich gewässert und dann mit Hämatoxylin-Eosin gefärbt. Die Herstellung der autoradiographischen Präparate erfolgte nach der „stripping-film"-Methode von $\mathrm{Pelc} \mathrm{c}^{26}$, entspr. der Beschreibung von $\mathrm{He} \mathrm{r} \mathrm{z}{ }^{27}$. Die benutzten Filme wurden von Ilford Ltd. und K o d a k Ltd. bezogen.

III. E r g e b n i s s e : Zunächst wurde geprüft, wie vollständig die Extraktion der säurelöslichen Phosphate und der Phospholipoide bei der üblichen Gewebsbehandlung ist. Untersuchung der Carnoyschen Lösung nach der Fixation ergab, daß 5 bis $25 \%$ der Gesamtaktivität aus dem Gewebe heraus-

20 H. S. di S t e f a n o, Science [Washington] 115, 316 [1952].

21 N. M. Sulkin u. A. Kuntz, Proc. Soc. exp. Biol. Med. 73, 413 [1950].

22 H. S t i c h, Z. Naturforschg. 6 b, 259 [1951].

23 E. R. F i s h e r, Stain Technol. 28, 9 [1953].

24 N. M. S u lki n, Proc. Soc. exp. Biol. and Med. 78, 32 [1951].

$25 \mathrm{~K}$. N e u m a n n, Grundriß der Gefriertrocknung; Musterschmidt-Verlag, Göttingen 1952.

26 S. R. P e l c, Nature [London] 160, 749 [1947].

27 R. H. H e r z, Med. Radiogr. Photogr. 26, 46 [1950]. 
gelöst wurden. Dabei hängt das Ausmaß der Extraktion von verschiedenen Faktoren ab, z. B. der Zeitspanne zwischen Injektion des Radio-Phosphors und Tötung des Versuchstieres, Art und Größe des Gewebsstückes usw. Gewebe mit hohem Nucleinsäure-Umsatz (Tumoren, Milz) zeigten meist geringere prozentuale Aktivitätsverluste als die Leber, aus welcher bis $\mathrm{zu} 25 \%$ ausgewaschen wurde. Im 96proz. Alkohol, den wir zur Herstellung histologischer Präparate der Carnoyschen Lösung folgen ließen. wurde meist nur um 1\% der Aktivität abgegeben, im dann folgenden absoluten Alkohol bei allen Versuchen weniger als $1 \%$. In Methylbenzoat, Benzol und Paraffin ging praktisch keine Radioaktivität verloren.

Nach dem Schneiden des Gewebes und Entparaffinieren der Schnitte fanden sich im Xylol nur Spuren an Radioaktivität. Die Aktivitätsverluste während der weiteren Behandlung sind aus Tab. 1 zu ersehen. Für eine vollständigere Extraktion der säurelöslichen Phosphat-Fraktion ist noch ein mehrfaches Spülen der Schnitte in 10-proz. Trichloressigsäure (je 5 bis $10 \mathrm{~min}$ ) erforderlich. Danach kam es zu keinen merklichen Aktivitätsverlusten mehr, wenn die Schnitte bei Zimmertemperatur in annähernd neutralen wässerigen Lösungen über nicht zu lange Zeit (1 Stde.) exponiert wurden.

Im weiteren wurden zunächst die Hydrolyseverfahren mit Perchlorsäure und $\mathrm{KOH}$ geprüft, da es wünschenswert schien, ohne die schwer zu beschaffende Ribonuclease auszukommen. Es ergab sich dabei jedoch, daß es mit diesen Verfahren nicht möglich ist, die PNS vollständig aus Gewebsschnitten zu extrahieren, ohne zugleich die DNS anzugreifen und teilweise mit herauszulösen. Die Schonung der DNS ist noch am größten, wenn zur PNS-Hydrolyse mit 10-proz. Perchlorsäure bei tieferer Temperatur entsprechend längere Zeit (bis zu $24 \mathrm{Stdn}$. bei $+4^{\circ} \mathrm{C}$ ) exponiert wird. Durch höhere Temperatur $\left(25^{\circ}\right.$ und $37^{\circ} \mathrm{C}$ ) wird zwar die Hydrolyse der PNS beschleunigt, doch führt dies zugleich zu stärkeren Verlusten von DNS. Dasselbe gilt für die alkalische Hydrolyse nach $\mathrm{S} u l \mathrm{kin}^{24}$, bei der die gleichzeitig erfolgende Proteolyse außerdem zu wenig schönen histologischen Präparaten führt.

Auf Grund dieser negativen Befunde wurde nun versucht, die PNS mit kristallisierter Ribonuclease enzymatisch zu hydrolysieren. Für die Angriffsmöglichkeit dieses Fermentes spielt die vorangegangene Fixierung des Gewebes eine maßgebliche Rolle ${ }^{14}$.
Fixierung in Carno y scher Lösung erwies sich als gut geeignet, wie schon von verschiedenen Autoren angegeben wurde; günstiger war die Fixierung durch Gefriertrocknung 10, 25 .

Auch mit der Ribonuclease-Behandlung gelangten wir jedoch nicht an das Ziel, aus dem Gewebe außer der DNS alle übrigen Phosphat-Fraktionen zu extrahieren. Zwar fällt chemisch-analytisch der in den Zellen zurückbleibende Rest nicht so sehr ins Gewicht, wohl aber bezüglich seiner Radioaktivität, die besonders bei der Leber ein Vielfaches der Strahlungsemission der DNS erreicht. Auch eine Nachbehandlung der Schnitte mit 10-proz. Perchlorsäure brachte keinen wesentlichen Gewinn; wirksamer war eine auf die oben beschriebene Prozedur folgende kurzzeitige Exposition der Schnitte in 0,1-n. $\mathrm{KOH}$, die hinwiederum gleichzeitig DNS mitextrahierte. Ein Beispiel solcher Versuche zeigt Tab. 2. Die letzte Spalte dieser Tabelle demonstriert eindringlich, in welchem Maße die Radioaktivität der Phosphoproteine die der DNS überlagert. Wenn selbst nach Ribonuclease-Einwirkung und Extraktion der säurelöslichen Phosphat-Fraktion und der P-Lipoide im Gewebsschnitt der Milz nur 52\%, in Schnitten der Leber sogar nur $11 \%$ der verbleibenden Aktivität von der DNS herrührt und der Rest von anderen radioaktiven Phosphorfraktionen, kann von einer selektiven Autoradiographie der DNS keine Rede sein.

Nachdem sich dies herausgestellt hatte, versuchten wir, durch andere Maßnahmen die Verhältnisse günstiger zu gestalten. Das gelang durch Einführung einer größeren Zeitspanne zwischen Injektion des Radio-Phosphors und der Tötung der Tiere. Dem liegt folgende Überlegung zugrunde: Die schwerwiegendste Fehlerquelle beim Versuch, selektiv die DNS autoradiographisch darzustellen, ist die hohe Aktivität der Phospho-Proteine. Die intensive Aufnahme von Radio-Phosphor durch diese Fraktion und die übrigen Substanzen läßt darauf schließen, daß diese sehr schnell vom Gewebe neu gebildet werden; dieser sehr lebhaften Synthesetätigkeit entspricht naturgemäß auch ein rascher Wiederabbau, bei dem ebenfalls die eingebauten Radio-Atome mit abgegeben werden. Andererseits hat die DNS einen wesentlich langsameren Umsatz als die PhosphoProteine und nimmt somit zwar anfangs weniger Radio-Phosphor auf, hält diesen aber auch dann noch fest, wenn die Phospho-Proteine ihre Aktivität schon weitgehend verloren haben, So ist, wenn auch die 


\begin{tabular}{|c|c|c|c|c|c|c|c|}
\hline Behandlung des Gewebes & Organ & $\begin{array}{l}\text { Unters. } \\
\text { Fraktion }\end{array}$ & $\frac{\text { mg P }}{\text { g Gew. }}$ & $\frac{\text { Aktiv.* }}{\text { g Gew. }}$ & $\begin{array}{l}\text { Spezif. Akt. } \\
\left(\frac{\text { Aktiv. }}{\text { mg P }}\right)\end{array}$ & $\begin{array}{c}\text { Restaktiv. } \\
\text { PNS+PP } \\
\text { bzw. DNS** } \\
{[\%]}\end{array}$ & $\begin{array}{c}\text { Anteil der } \\
\text { DNS a. d. } \\
\text { verbliebenen } \\
\text { Gewebsaktiv. } \\
{[\%]}\end{array}$ \\
\hline \multirow{2}{*}{ Kontrollen } & Milz & $\underset{\mathrm{PNS}+\mathrm{PP}}{\mathrm{PNS}}$ & - & $\begin{array}{r}110600 \\
48000\end{array}$ & - & $\begin{array}{l}100 \\
100\end{array}$ & $\overline{29}$ \\
\hline & Leber & $\begin{array}{c}\mathrm{PNS}+\mathrm{PP} \\
\mathrm{DNS}\end{array}$ & $\begin{array}{l}0,650 \\
0,207\end{array}$ & $\begin{array}{r}191000 \\
12800\end{array}$ & $\begin{array}{r}293000 \\
61700\end{array}$ & $\begin{array}{l}100 \\
100\end{array}$ & $\overline{6,3}$ \\
\hline \multirow{2}{*}{ Ribonuclease } & Milz & $\begin{array}{c}\mathrm{PNS}+\mathrm{PP} \\
\mathrm{DNS}\end{array}$ & - & $\begin{array}{l}45000 \\
48000\end{array}$ & - & $\begin{array}{r}41 \\
100\end{array}$ & $\overline{51,6}$ \\
\hline & Leber & $\begin{array}{c}\mathrm{PNS}+\mathrm{PP} \\
\mathrm{DNS}\end{array}$ & $\begin{array}{l}0,164 \\
0,207\end{array}$ & $\begin{array}{r}115000 \\
12800\end{array}$ & $\begin{array}{r}705000 \\
61700\end{array}$ & $\begin{array}{r}58 \\
100\end{array}$ & $\overline{10}$ \\
\hline $\begin{array}{c}\text { Ribonuclease } \\
+0,1-n \cdot \operatorname{KOH}\left(12^{\prime}\right)\end{array}$ & Leber & $\begin{array}{c}\mathrm{PNS}+\mathrm{PP} \\
\mathrm{DNS}\end{array}$ & $\begin{array}{l}0,065 \\
0,098\end{array}$ & $\begin{array}{r}59200 \\
6900\end{array}$ & $\begin{array}{r}903000 \\
71000\end{array}$ & $\begin{array}{l}31 \\
54\end{array}$ & $\overline{10,4}$ \\
\hline $\begin{aligned} & \text { Ribonuclease } \\
+ & 0,1-n \cdot \operatorname{KOH}\left(25^{\prime}\right)\end{aligned}$ & Leber & $\begin{array}{c}\mathrm{PNS}+\mathrm{PP} \\
\mathrm{DNS}\end{array}$ & $\begin{array}{l}0,061 \\
0,094\end{array}$ & $\begin{array}{r}53000 \\
4700\end{array}$ & $\begin{array}{r}870000 \\
50000\end{array}$ & $\begin{array}{l}28 \\
37\end{array}$ & $\overline{8,2}$ \\
\hline
\end{tabular}

Tab. 2. Übersicht über die Auswirkung verschiedener Methoden, die Pentosenucleinsäure aus Gewebsschnitten zu extrahieren. Die Zahlen stellen jeweils Mittelwerte von 3 oder 4 Parallelanalysen dar. Das zur Untersuchung verwandte Gewebe wurde einer Ratte entnommen (Gewicht 170 g), der 6 Stdn. vor der Tötung 1 mC Radio-Phosphor als Orthophosphat intraperitoneal injiziert worden war. Fixierung in Carnoyscher Lösung.

chemische mißlingt, eine "biophysikalische“ Ausschaltung der störenden radioaktiven Phospho-Proteine weitgehend möglich. Die Zeitspanne zwischen der Verabfolgung eines Radio-Isotops und der Tötung des Versuchstieres hat also auf das Verhältnis der Aktivitäten verschiedener Gewebsfraktionen zueinander einen maßgeblichen Einfluß ${ }^{9}$. Dies zeigt am Beispiel des Crocker-Sarkoms die Tab. 3.

\begin{tabular}{|c|c|}
\hline $\begin{array}{c}\text { Zeitspanne zwischen }{ }^{32} \mathrm{P}- \\
\text { Injektion und Tötung }\end{array}$ & $\begin{array}{c}\text { Anteil der DNS an der ver- } \\
\text { bliebenen Gewebsaktivität } \\
{[\%]}\end{array}$ \\
\hline 4 Stdn. & 29 \\
36 Stdn. & 78 \\
5 Tage & 85 \\
\hline
\end{tabular}

Tab. 3. Anteil der DNS an der Radioaktivität von Gewebsschnitten des Mäusesarcoms 180 (Crocker) nach Ribonuclease-Behandlung und Perchlorsäure-Extraktion.

Während also bei Tötung 4 Stdn. nach der Injektion für die Aktivität der DNS im rasch wachsenden Crocker-Sarkom der Fehler durch die Phospho-

* Die Aktivität ist angegeben in Zählrohrstößen pro Minute.

** Bei diesen Werten ist nicht berücksichtigt, daß durch die Carnoy-Fixation beide Nucleinsäure-Fraktionen bereits Verluste erlitten haben $\mathbf{1 0}$.
Proteine noch $+245 \%$ betrug, verringerte er sich nach 36 Stdn. auf $+28 \%$, nach 5 Tagen auf $+19 \%$.

IV. Diskussion: In der Originalarbeit von Schmidt und Thannhause ${ }^{\overline{ }}{ }^{\top}$ wurde noch angegeben, daß nach der Extraktion der säurelöslichen Phosphat-Fraktion und der Phospholipoide im Gewebe an phosphor-haltigen Substanzen im wesentlichen nur noch die beiden Nucleinsäure-Fraktionen vorlägen; die daneben vorhandenen Phospho-Proteine seien in den meisten Geweben praktisch zu vernachlässigen. Doch wurde schon von $D$ avids o ${ }^{4}$ eindringlich darauf hingewiesen, daß bei Untersuchungen mit Radio-Phosphor die Phospho-Proteine keinesfalls belanglos seien; da sie besonders viel ${ }^{32} \mathrm{P}$ aufnehmen und so zu beträchtlichen Fehlern bei der Beurteilung der spezifischen Aktivität der PNS führen können. Die hohe Stoffwechselaktivität der Phospho-Proteine wurde jetzt durch neuere Untersuchungen von $\mathrm{Johns}$ on ${ }^{28}$ bestätigt. Mit Hilfe der Papierelektrophorese zeigten $\mathrm{Davids}$ on und S melli e ${ }^{5}$, daß außer den Phospho-Proteinen noch weitere - chemisch noch nicht näher analysierte Phosphatverbindungen im Gewebe verbleiben, die ebenfalls alle auch sehr viel ${ }^{32} \mathrm{P}$ aufnehmen. Diese

28 R. M. Johnson u. S. Albert, J. biol. Chemistry 200, 335 [1953]. 
Fraktionen bleiben nach der Extraktion der säurelöslichen Phosphate und der Phospholipoide in den Gewebsschnitten zurück und tragen bei der anschlieBenden Autoradiographie ganz wesentlich zu der Schwärzung der Photoemulsion bei. Es ist daher eine irrige Vorstellung, daß bei üblicher histologischer Prozedur die Restaktivität des Radio-Phosphors im Gewebe ausschließlich den Nucleinsäuren zuzuschreiben sei. Noch größer wird dieser Fehler, wenn man zur selektiven Darstellung der DNS die PNS extrahiert. Die im Gewebe zurückbleibende genannte Fraktion überdeckt nun mit ihrer hohen Aktivität fast völlig die der DNS. Dies wirkt sich in den einzelnen Organen verschieden aus, am schwerwiegendsten in solchen mit geringem DNS-Umsatz.

Doch macht nicht nur diese Fehlerquelle die selektive autoradiographische Darstellung der DNS zu einer mißlichen Sache. Ein weiteres Problem stellt die vollständige Extraktion der PNS unter vollkommener Erhaltung der DNS dar. Zwar erlaubte die angewandte Untersuchungstechnik nicht, das Ausmaß der Ribonuclease-Wirkung exakt zu bestimmen. Nach den Zahlen der Tab. 2 und weiteren Befunden ist es aber wahrscheinlich, daß nach der Einwirkung der Ribonuclease noch ein Rest an PNS im Gewebe zurückbleibt. Dieser läßt sich wohl durch Nachbehandlung mit 10-proz. Perchlorsäure oder 0,1-n. KOH verringern, aber nicht beseitigen. Während die Perchlorsäure offenbar die Phospho-Proteine und die übrigen störenden Substanzen nicht angreift, lassen sich diese durch 0,1-n. KOH teilweise aus dem Gewebe herauslösen. Praktisch bedeutet dies jedoch keinen Gewinn, da hierbei gleichzeitig DNS verlorengeht (als Beispiel die Werte der Tab. 2).

Angesichts dieser Befunde erhebt sich die Frage, ob es überhaupt möglich ist, den DNS-Umsatz im Gewebe autoradiographisch zu untersuchen. Da eine einigermaßen quantitative Extraktion der störenden phosphat-haltigen Substanzen offenbar nicht durchführbar ist, könnte man daran denken, mit Hilfe kristalliner Desoxyribonuclease auch noch die DNS zu extrahieren; der Unterschied im Schwärzungsgrad der Autoradiographien müßte dann nach RibonucleaseBehandlung und anschließender Einwirkung von Desoxyribonuclease ausreichend sein, um auf den DNS-Umsatz schließen zu können. Einfacher ist der von uns beschriebene Ausweg, die Zeitspanne zwischen Injektion und Tötung des Versuchstieres größer $\mathrm{zu}$ wählen. Auf diese Weise wird wenigstens bei Organen mit großer Mitoserate und dementsprechend hohem DNS-Umsatz der Fehler durch die PhosphoProteine und die übrigen störenden Substanzen zwar nicht beseitigt, jedoch so klein, daß für qualitative Beurteilung eine autoradiographische Darstellung der DNS möglich wird.

Neben diesen histochemischen Problemen besteht jedoch noch eine grundsätzliche Schwierigkeit. Die zur autoradiographischen Darstellung der Nucleinsäuren erforderlichen Dosen an ${ }^{32} \mathrm{P}$ sind recht hoch und führen in dem untersuchten Organ zu einer beträchtlichen Strahlendosis. Als Folge der Strahlenwirkung wird in erster Linie die Neubildung der DNS gehemmt, also gerade der Substanz, deren Stoffwechsel untersucht werden soll. Als kritische Dosis wird im allgemeinen 2-3 $\mu \mathrm{C} / \mathrm{g}$ Körpergewicht angegeben, die bei autoradiographischer Darstellung der DNS jedoch meist nicht ausreicht.

Die autoradiographische Untersuchung des normalen DNS-Stoffwechsels mit Hilfe von Radio-Phosphor ist demnach mit so viel Fehlerquellen belastet, daß so erhobene Befunde nur mit großer Zurückhaltung verwendet werden können. Als Ergänzung zu Untersuchungen am Gewebshomogenat bei gestörtem DNS-Umsatz durch Röntgenstrahlen scheint uns jedoch die Autoradiographie geeignet, wesentliche qualitative Hinweise zu geben. 